\title{
Mechanical optimization of superconducting cavities in continuous wave operation
}

\author{
Sam Posen* and Matthias Liepe \\ Cornell Laboratory for Accelerator-Based Sciences and Education, Cornell University, Ithaca, New York 14853, USA
}

(Received 1 December 2011; published 14 February 2012)

\begin{abstract}
Several planned accelerator facilities call for hundreds of elliptical cavities operating cw with low effective beam loading, and therefore require cavities that have been mechanically optimized to operate at high $Q_{L}$ by minimizing $d f / d p$, the sensitivity to microphonics detuning from fluctuations in helium pressure. Without such an optimization, the facilities would suffer either power costs driven up by millions of dollars or an extremely high per-cavity trip rate. ANSYS simulations used to predict $d f / d p$ are presented as well as a model that illustrates factors that contribute to this parameter in elliptical cavities. For the Cornell Energy Recovery Linac (ERL) main linac cavity, $d f / d p$ is found to range from 2.5 to $17.4 \mathrm{~Hz} / \mathrm{mbar}$, depending on the radius of the stiffening rings, with minimal $d f / d p$ for very small or very large radii. For the Cornell ERL injector cavity, simulations predict a $d f / d p$ of $124 \mathrm{~Hz} / \mathrm{mbar}$, which fits well within the range of measurements performed with the injector cryomodule. Several methods for reducing $d f / d p$ are proposed, including decreasing the diameter of the tuner bellows and increasing the stiffness of the enddishes and the tuner. Using measurements from a Tesla Test Facility cavity as the baseline, if both of these measures were implemented and the stiffening rings were optimized, simulations indicate that $d f / d p$ would be reduced from $\sim 30 \mathrm{~Hz} / \mathrm{mbar}$ to just $2.9 \mathrm{~Hz} / \mathrm{mbar}$, and the power required to maintain the accelerating field would be reduced by an order of magnitude. Finally, other consequences of optimizing the stiffening ring radius are investigated. It is found that stiffening rings larger than $70 \%$ of the iris-equator distance make the cavity impossible to tune. Small rings, on the other hand, leave the cavity susceptible to plastic deformation during handling and have lower frequency mechanical resonances, which is undesirable for active compensation of microphonics. Additional simulations of Lorentz force detuning are discussed, and the results are compared to measurements on the ERL injector cavities.
\end{abstract}

DOI: 10.1103/PhysRevSTAB.15.022002

PACS numbers: 29.20.Ej, 02.70.Dh, 46.40.-f

\section{BACKGROUND}

Historically, the mechanical design of elliptical superconducting particle accelerator cavities has been optimized mainly for pulsed machines, and has therefore focused on the reduction of Lorentz force detuning (LFD). On the other hand, for low $\beta$ cavities, significant effort has been put forward to optimizing the mechanical design for minimizing power requirements when running $\mathrm{cw}$ with high loaded quality factor (see, for example, [1-5]). However, new $\mathrm{cw}$ particle accelerator designs are pushing elliptical cavities into operating regimes with low or no effective beam loading and thus a high optimal loaded $Q$. A selection of these accelerators with their operating parameters is shown in Table I. The mechanical design of elliptical cavities must be reoptimized to make them effective in these machines.

\footnotetext{
*sep93@cornell.edu
}

Published by the American Physical Society under the terms of the Creative Commons Attribution 3.0 License. Further distribution of this work must maintain attribution to the author(s) and the published article's title, journal citation, and DOI.

\section{INTRODUCTION}

The rf power $P$ required to maintain a constant accelerating voltage $V_{\text {acc }}$ in a superconducting cavity with geometric shunt impedance $R / Q$ (accelerator definition) and external quality factor $Q_{\mathrm{ext}}$ is given by Eq. (1) (for $Q_{\mathrm{ext}} \gg$ $Q_{0}$, i.e., strongly overcoupled case):

$$
\begin{aligned}
P= & \frac{V_{\mathrm{acc}}^{2}}{4 \frac{R}{Q} Q_{\mathrm{ext}}}\left[\left(1+\frac{R}{Q} Q_{\mathrm{ext}} \frac{I_{b}}{V_{\mathrm{acc}}} \cos \phi_{b}\right)^{2}\right. \\
& \left.+\left(2 Q_{\mathrm{ext}} \frac{\Delta f}{f_{0}}+\frac{R}{Q} Q_{\mathrm{ext}} \frac{I_{b}}{V_{\mathrm{acc}}} \sin \phi_{b}\right)^{2}\right]
\end{aligned}
$$

Here $I_{b}$ is the average beam current, $\phi_{b}$ is the accelerating phase, $f_{0}$ is the cavity frequency, and $\Delta f$ is the cavity detuning. The optimal external quality factor to minimize $P$ is given by Eq. (2):

$$
Q_{\mathrm{opt}}=\left[\left(\frac{R}{Q} \frac{I_{b}}{V_{\mathrm{acc}}}\right)^{2}+\left(\frac{2 \Delta f}{f_{0}}\right)^{2}+2 \frac{R}{Q} \frac{I_{b}}{V_{\mathrm{acc}}} \frac{2 \Delta f}{f_{0}} \sin \phi_{b}\right]^{-1 / 2} .
$$

Figure 1 plots the required rf power according to Eq. (1) assuming optimal $Q_{\text {ext }}$ from Eq. (2) and $\phi_{b}=0$ using parameters from the Cornell Energy Recovery Linac (ERL) main linac. 
TABLE I. A selection of cw linacs under design with small beam loading. Detuning overhead refers to the maximum detuning that can be tolerated in a cavity at the design gradient before running out of rf power. Data are from [6-9].

\begin{tabular}{lccc}
\hline \hline Facility & $\begin{array}{c}\text { Cornell ERL } \\
\text { 7-cell elliptical }\end{array}$ & $\begin{array}{c}\text { KEK ERL } \\
\text { 9-cell elliptical }\end{array}$ & $\begin{array}{c}\text { Project X } \\
\text { Mostly 5-cell elliptical }\end{array}$ \\
\hline$\beta$ & 1 & 1 & $0.61 / 0.9$ \\
No. of cavities required & $\sim 400$ & 8 (prototype) $\sim 200$ (full ERL) & $42 / 152$ \\
Gradient $[\mathrm{MV} / \mathrm{m}]$ & 16.2 & $\sim 15$ & $16.6 / 18.7$ \\
Effective current $[\mathrm{mA}]$ & 0 & 0 & 1 \\
Frequency $[\mathrm{GHz}]$ & 1.3 & 1.3 & 0.65 \\
$Q_{L}$ & $6.5 \times 10^{7}$ & $1 \times 10^{7}-4 \times 10^{7}$ & $3.3 \times 10^{7} / 3.4 \times 10^{7}$ \\
Full bandwidth $[\mathrm{Hz}]$ & 20 & $32-130$ & $20 / 19$ \\
Detuning overhead $[\mathrm{Hz}]$ & 20 & 100 & 20 \\
\hline \hline
\end{tabular}

From Fig. 1 it is clear that the minimum rf power depends strongly on the cavity detuning when the effective beam current is small. Under these conditions, small perturbations on the order of tens of $\mathrm{Hz}$ can be significant. Even microphonics, external vibrations that change the frequency of the cavity, can greatly change the required rf power.

If the level of microphonics detuning is underestimated, and the cavity is detuned so much that there is not enough power available to maintain a constant field, one of two things may happen. If the detuning is negative, then the

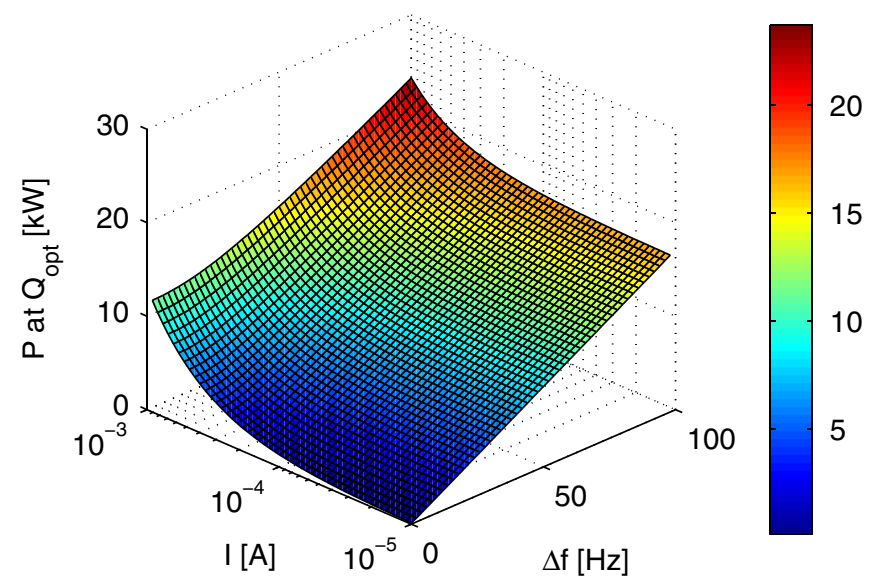

FIG. 1. Power required to maintain a constant accelerating gradient as a function of current and detuning. Parameters from the Cornell ERL main linac are used.

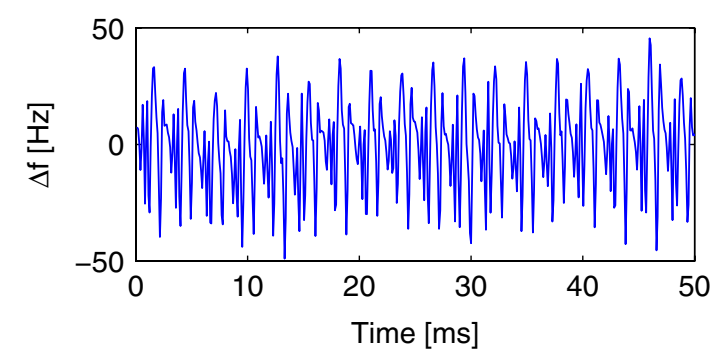

FIG. 2. Detuning of a Cornell ERL injector cavity in the presence of microphonics.
LFD will increase the frequency as the field decreases and help it to recover. However, if the detuning is positive, then the LFD will further detune the cavity, and it will trip. A high trip rate is unacceptable for most superconducting radiofrequency (SRF) systems, especially in an x-ray user facility, where researchers require continuous beam.

As Fig. 1 shows, higher levels of microphonics require more rf power per cavity. The cost of this additional $\mathrm{rf}$ power is high, especially in machines with hundreds of cavities. This includes not just operational costs for grid power, but also infrastructure costs incurred by larger rf power sources.

Microphonics detuning can be reduced using active compensation from fast piezoelectric actuator tuners, but their effectiveness and bandwidth is limited by mechanical resonances in the cavity and cryomodule. The only reliable method of reducing power requirements is to passively minimize microphonics detuning. One source of microphonics is ground vibration, but studies [10] have shown that these vibrations can be decoupled from the cavities through careful cryomodule design. Therefore fluctuations in the helium bath pressure are most likely the main source of microphonics detuning. The detuning of a cavity in the Cornell ERL injector prototype is shown in Fig. 2. Microphonics detuning is the product of the helium pressure fluctuation $\Delta p$-the maximum value of which should be made as low as possible through optimization of the cryogenic system-and the cavity pressure sensitivity $d f / d p$, i.e. $\Delta f=(d f / d p) \Delta p . d f / d p$ depends on the cavity size and shape, the number of cells, the cavity material thickness, the stiffening rings, the helium tank, and the tuner.

\section{CASE STUDY: THE CORNELL ERL}

A detailed study of the pressure sensitivity $d f / d p$ of the Cornell ERL cavities is presented in this paper. The Cornell ERL requires about 4007 -cell cavities in the main linac section, each with its own $5 \mathrm{~kW}$ power supply. The ERL 7-cell is an elliptical cavity with large beam tubes for higher order mode damping, and stiffening rings between each cell and after both end cells. In the iteration of 


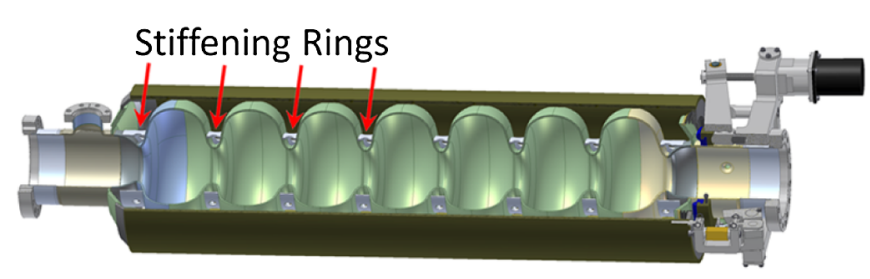

FIG. 3. Section view of a CAD model of a Cornell ERL main linac cavity. In the model shown, the cavity has stiffening rings at the same radius used in ILC cavities.

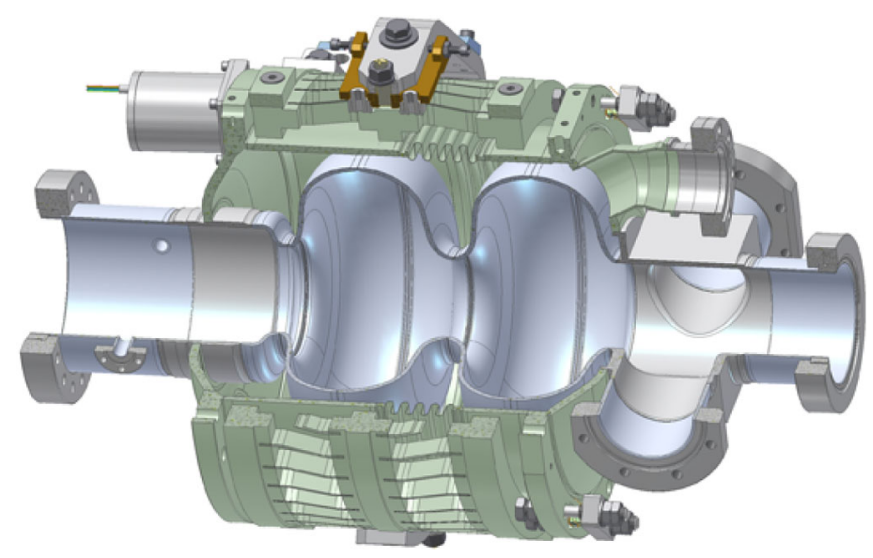

FIG. 4. Section view of a CAD model of a Cornell ERL injector cavity.

the design under study in this paper, the cavity has two $\mathrm{NbTi}$ enddishes, one of which is rigidly welded to the $\mathrm{Ti}$ helium tank, while the other is separated from the tank by a bellows, allowing the cavity length to be adjusted by a Saclay I-type tuner [11]. A computer-aided design (CAD) model of the cavity is presented in Fig. 3.

This study is meant to optimize the radius of the 7-cell cavity's stiffening rings and the design of its helium tank with the goal of minimizing $d f / d p$. The specifications of the tuner may also be influenced. The shape of the cavity has already been finalized based on rf requirements [12]. This study focuses on the ERL main linac cavity, but the conclusions are generally applicable to other elliptical cavity designs for $\mathrm{cw}$ operation with small effective beam current.

Simulations of the 7-cell cavity cannot be directly compared with measurements in this paper, as the first ERL cavity is in the production stage as of the date of writing. However, five 2-cell cavities currently in operation in the ERL injector cryomodule are available for testing [13,14]. These cavities are not optimized for low microphonics, but they should provide a means of comparison between simulations and measurements, and thus of verifying the simulation tools used here. The 2-cell cavities use an INFN blade tuner [15], ${ }^{1}$ and have helium tanks with a bellows in

\footnotetext{
${ }^{1}$ This is based on the Istituto Nazionale di Fisica Nucleare blade tuner.
}

the middle to allow the length to change. The cavityhelium tank-tuner assembly is shown in Fig. 4.

\section{SIMULATIONS AND MEASUREMENT}

The influence of the stiffening ring radius and the helium tank design on $d f / d p$ can be studied through coupled structural-high frequency electromagnetic simulation. Using the engineering software package ANSYS [16], simulations were developed [17] that calculate the fundamental accelerating mode frequency of a cavity, apply a pressure load to deform it, and then calculate the new fundamental accelerating mode frequency. For maximum accuracy, the complete cavity assembly was used in the simulations, including the helium tank and the tuner. The mesh uses tetrahedral elements with midside nodes. The same mesh is used for the entire simulation, switching the element types between structural and high frequency for different analyses, and updating the mesh after determining the deformation due to the pressure load rather than remeshing. This greatly improves accuracy without requiring a very dense and computationally expensive mesh.

A mesh from the simulations is shown in Fig. 5. The simplified tuner model used in these simulations is visible as the rigid plate and bellows-like part. The material properties of the bellows part were modified to give it the stiffness reported for the Saclay I tuner, $100 \mathrm{kN} / \mathrm{mm}$ [11]. A similar bellows part was used to simulate the blade tuners of the injector cavities which have a reported [15] stiffness of $31 \mathrm{kN} / \mathrm{mm}$ (a 25\% increase in stiffness is assumed for cryogenic temperatures). Material properties for $\mathrm{Nb}, \mathrm{NbTi}$, and $\mathrm{Ti}$ were obtained from [18]. The accuracy of the simulations depends strongly on the accuracy of the material properties.

Measurements were carried out on the ERL injector cryomodule cavities. $d f / d p$ was measured by slowly varying the helium pressure by several mbar and observing the change in cavity frequency. The LFD of the cavities was measured by comparing the frequency at low accelerating gradients to the frequency at high gradients.

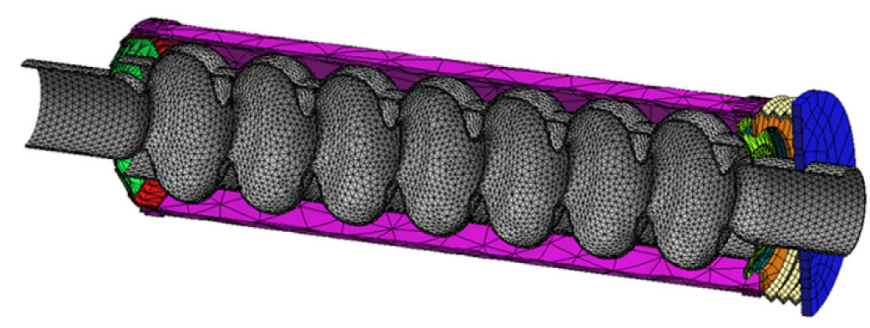

FIG. 5. ANSYS mesh of the ERL 7-cell cavity with helium tank. The tan bellows-like part on the right is the simplified tuner. It is supported by the rigid blue plate. In this model, the stiffening rings between the cells have a $155 \mathrm{~mm}$ inner diameter. 


\section{V. $d f / d p$ RESULTS}

The simulation results for the 7-cell cavity are presented in Fig. 6. $d f / d p$ was calculated as a function of stiffening ring radius for the nominal $100 \mathrm{kN} / \mathrm{mm}$ tuner stiffness and $3 \mathrm{~mm}$ material thickness, as well as values close to nominal. The stiffening ring radial position is presented as a fraction of the iris-equator distance, $\left(r_{\text {ring }}-r_{\text {iris }}\right) /$ $\left(r_{\text {equator }}-r_{\text {iris }}\right)$.

In every case, the $d f / d p$ trends in Fig. 6 show the same pattern: a maximum at about $25 \%$ of the iris-equator distance, and a significant decrease as the radius grows or shrinks. The minimum $d f / d p$ occurs when the stiffening rings are at the equator. With $2 \mathrm{~mm}$ walls, $d f / d p$ even becomes negative, indicating that the value is in fact zero for some stiffening ring radius. For the nominal values, there are two optimal locations for stiffening rings to minimize $d f / d p$ : one is to have no stiffening rings at all; the other is to have stiffening rings with a very large radius. As discussed later, there are limitations on how large the stiffening rings can be, and taking this into account, the values of $d f / d p$ given by these two solutions are very close.

Increasing the tuner stiffness decreases $d f / d p$ for all stiffening ring radii, and the decrease is most dramatic for smaller radii. Decreasing the wall thickness makes the shape of the curve more pronounced-the maximal $d f / d p$ values become larger and the minimal ones become smaller. Depending on the scenario, it may be desirable to adjust the wall thickness to decrease $d f / d p$.

The International Linear Collider (ILC) cavity has a shape very similar to that of the ERL 7-cell. However, ILC cavities are optimized to reduce LFD, not microphonics detuning. As indicated in Fig. 6, the ILC stiffening

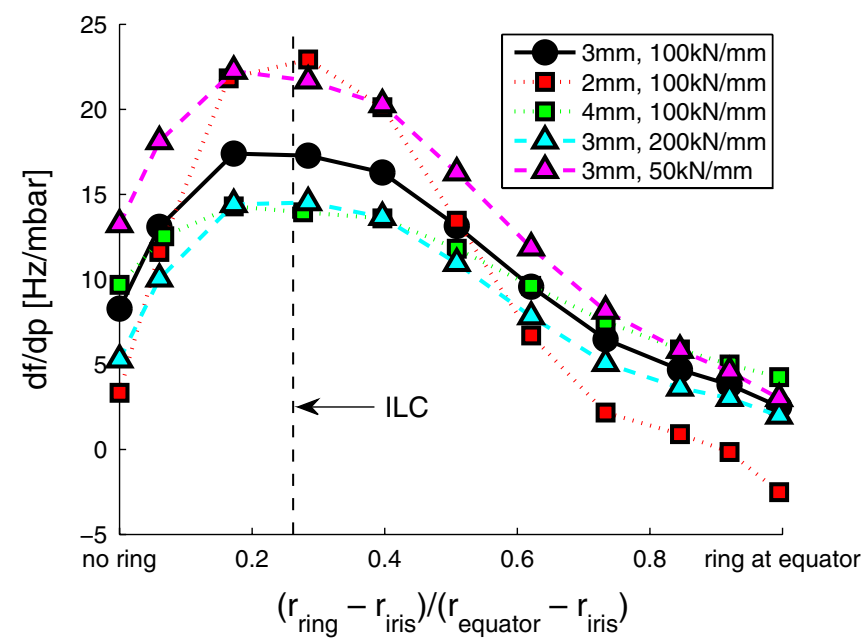

FIG. 6. $d f / d p$ of the 7-cell cavity for several wall thicknesses from 2 to $4 \mathrm{~mm}$ and tuner stiffnesses from 50 to $200 \mathrm{kN} / \mathrm{mm}$, as a function of the radial position of the stiffening ring, given as a fraction of the iris-equator distance [i.e. $\left(r_{\text {ring }}-r_{\text {iris }}\right) /$ $\left.\left(r_{\text {equator }}-r_{\text {iris }}\right)\right]$. rings lie very close to the maximum of $d f / d p$. In this location, they prevent deformation at the iris, decreasing LFD. On the other hand, under an increase in helium pressure, the negative frequency shift caused by iris deformation counters the positive frequency shifts caused by equator deformation and length increase. As a result, $d f / d p$ is smallest when the iris is free to deform.

From Fig. 6, simulation predicts a $d f / d p$ of $17 \mathrm{~Hz} / \mathrm{mbar}$ for the ERL 7-cell cavity with stiffening rings at $28 \%$ of the iris-equator distance. With these stiffening rings, the 7-cell would be very similar to the ILC 9-cell cavity, so the simulation prediction can be compared to measurements made on such cavities in operation at other labs. Values of 28 and $30 \mathrm{~Hz} / \mathrm{mbar}[19,20]$ were reported for cavities manufactured for Tesla Test Facility (TTF), and a value of $55 \mathrm{~Hz} / \mathrm{mbar}$ [21] was reported for HoBiCaT. The simulation prediction is about $40 \%$ lower than the smallest reported values, which is reasonable agreement, taking into account differences in the cavity and helium tank design, as well as how critically the simulation results rely on reported material properties, especially the stiffness of the tuner.

For the ERL 2-cell injector cavity, simulations predicted the $d f / d p$ to be $124 \mathrm{~Hz} / \mathrm{mbar}$. This is significantly higher than the 7-cell prediction, but the design of the 2-cell was not optimized for low $d f / d p$, as the injector operates at low loaded $Q$ for large effective beam loading. Measurements, averaged over all five cavities in the injector, are shown with the ANSYS prediction in Fig. 7. The measurement was repeated after changing the position of the tuner by +4000 steps (approximately $1 \%$ of the total range of the tuner), corresponding to a shift of approximately $-15 \mathrm{kHz}$, and by -4000 steps.

The error bars in Fig. 7 were derived from the significant spread in values between the five cavities. The order of

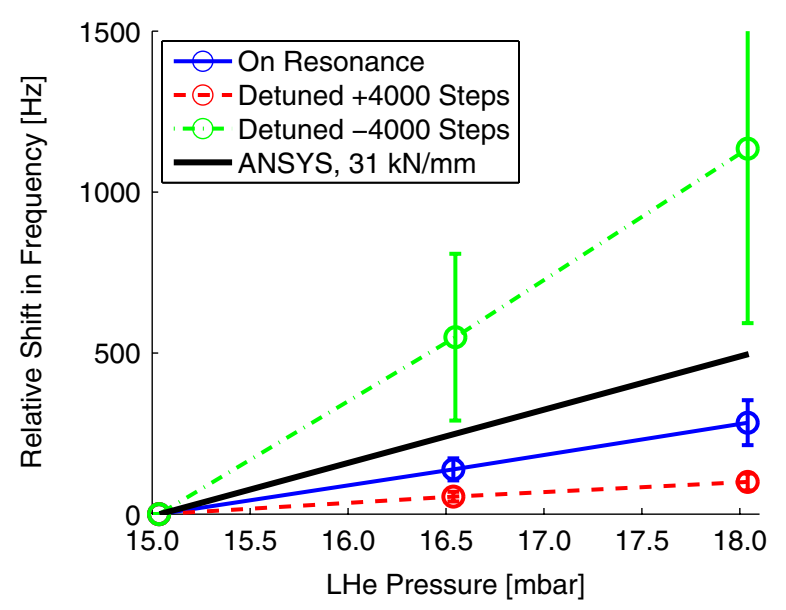

FIG. 7. Measured $\Delta f$ versus $\Delta p$ of the 2-cell cavities for different tuner positions. The prediction from ANSYS simulations is also shown, which assumes the stiffness of the tuner is $31 \mathrm{kN} / \mathrm{mm}$. 
TABLE II. Simulations used to find parameters from the $d f / d p$ model.

\begin{tabular}{lccc}
\hline \hline Parameter & Boundary conditions & Load & Result calculated \\
\hline$d f_{\text {shape }} / d p$ & Fix beam tubes & 1 bar helium pressure & $\Delta f$ \\
$K$ & Fix one beam tube & $1 \mathrm{kN}$ axial force to other beam tube & Length change \\
$d f / d L$ & Fix one beam tube & $1 \mu \mathrm{m}$ shift of other beam tube & $\Delta f$ \\
$d F / d p$ & Fix beam tubes & 1 bar helium pressure & Average restoring force \\
\hline \hline
\end{tabular}

magnitude difference in $d f / d p$ observed when changing only the position of the tuner shows that some factor is changing significantly with tuner position, likely the tuner's overall stiffness. Only one number was found reported for the measured stiffness of the blade tuner [15], and that number is used in the simulations, but the tuners on the ERL injector cavities may in fact be stiffer than this when on resonance. Taking into account the range of values obtained by varying the tuner position, the $d f / d p$ prediction from simulation agrees reasonably well with measurement.

Note that the 2-cell cavity $d f / d p$ prediction is a factor of about 15 times higher than that of the unstiffened 7-cell cavity. An analytical model can be used to understand this large difference in sensitivity.

\section{UNDERSTANDING CAVITY BEHAVIOR UNDER PRESSURE VARIATIONS USING AN ANALYTICAL MODEL}

The simulations discussed in the previous section are a useful tool for evaluating the $d f / d p$ of a particular cavityhelium tank-tuner configuration; however, they do not explain why two cavity-helium tank-tuner assemblies studied have $d f / d p$ values an order of magnitude apart, nor do they suggest a method for decreasing $d f / d p$ in a given assembly. To accomplish this, the individual factors contributing to $d f / d p$ must be understood in more detail.

First, one can isolate the $\Delta f$ contribution due to the change in length of the cavity from the contribution due to cell shape changes in the cavity at fixed length (as is done in [22]):

$$
\frac{d f}{d p}=\frac{d f_{\text {shape }}}{d p}+\frac{d f_{\text {length }}}{d p} .
$$

The length change can be deconstructed further using the length of the cavity $L$ and the force $F$ experienced by the cavity increasing its length:

$$
\frac{d f_{\text {length }}}{d p}=\frac{d f}{d L} \frac{d L}{d p}=\frac{d f}{d L} \frac{d L}{d F} \frac{d F}{d p}=\frac{d f}{d L} \frac{1}{K} \frac{d F}{d p} .
$$

Here $K$ is the stiffness of the cavity-helium tank-tuner assembly. ANSYS can calculate all these parameters using the procedures outlined in Table II.

$d f / d L$ depends on the shape and size of the cavity. The two cavities under study in this paper operate at the same frequency and have similar shapes, but different numbers of cells. One might therefore expect that $d f / d L$ depends linearly on this factor to first order [23].

$d f / d L$ is plotted as a function of stiffening ring radius in Fig. 8. Only one point is plotted for the injector cavity as its design does not include stiffening rings. The $d f / d L$ of the injector cavity is predicted by ANSYS to be 3.9 times that of the linac cavity without stiffening rings. This is very close to the expected factor of $7 / 2=3.5$ from the different number of cells.

In considering the longitudinal stiffness $K$ of the cavity assembly, one can think of it as a combination of springs in series and parallel, as shown in Fig. 9. This model can be used to better understand the contribution of each part to the stiffness of the overall assembly.

The models' predictions for $K$ are plotted in Fig. 10. The results from ANSYS simulations of the full model are also plotted. For the 7-cell, the full simulation shows excellent agreement with the simple combination of spring constants for small to medium ring radii, but the two start to differ at large stiffening ring radii. This is likely caused by double counting of the enddishes once the stiffening rings become large enough to connect with them in the cavity stiffness simulation. For the 2-cell without rings, the spring combination agrees well with the model.

In the spring model results in Fig. 10, the cavity is separated from the rest of the assembly to show its contribution. The contribution of each of the other parts is given in the sensitivity analysis in Table III. For this analysis, the stiffness of each part was decreased by $50 \%$ and the resulting effect on the stiffness of the assembly without the cavity was calculated.

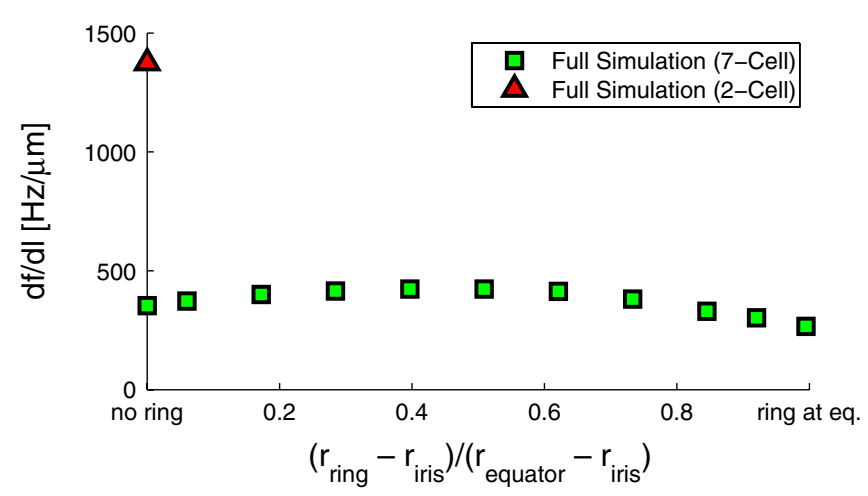

FIG. 8. $d f / d L$ of the Cornell ERL 7-cell main linac and 2-cell injector cavities. 


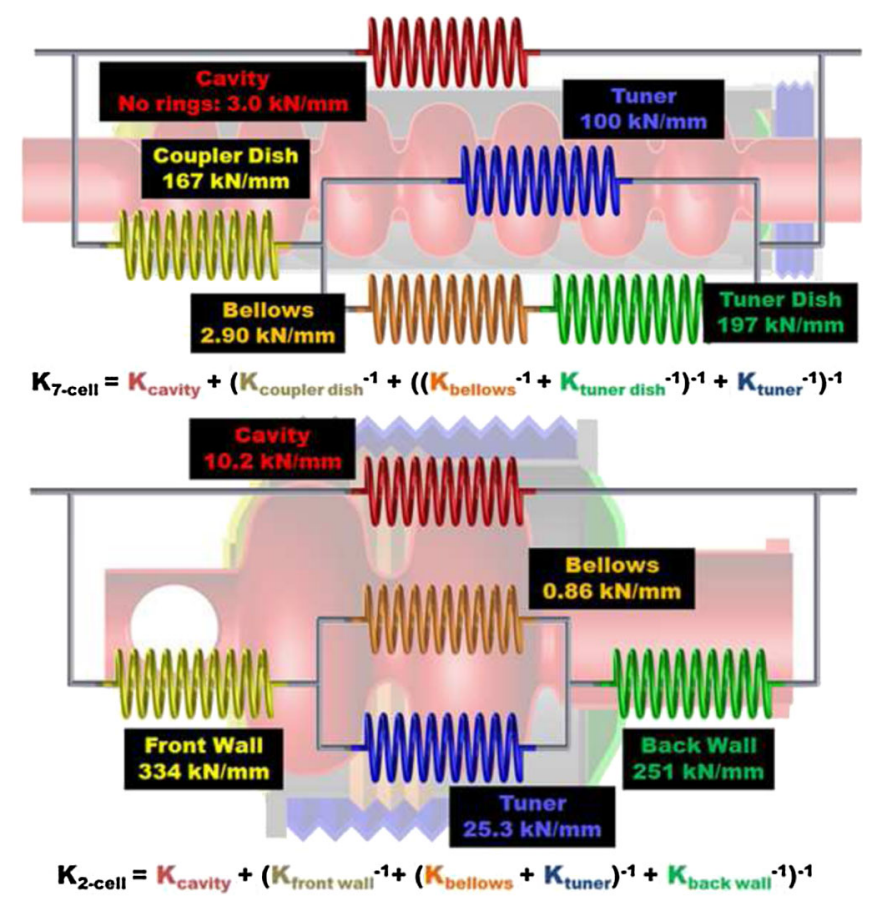

FIG. 9. Spring model of the longitudinal stiffness of the 7-cell assembly (above) and the 2-cell assembly (below).

Both cavity assemblies are very sensitive to the tuner stiffness. The factor of 4 difference between the Saclay tuner and the blade tuner gives the 7-cell assembly a much higher overall stiffness than the 2-cell assembly. The higher stiffness of the 2-cell cavity itself partially offsets the weakness of its blade tuner. It is also important to note that for the 7-cell assembly, the stiffness of the coupler enddish (i.e., the end wall of the helium tank opposite the end of the cavity with the tuner) has the second-largest impact on the overall stiffness.

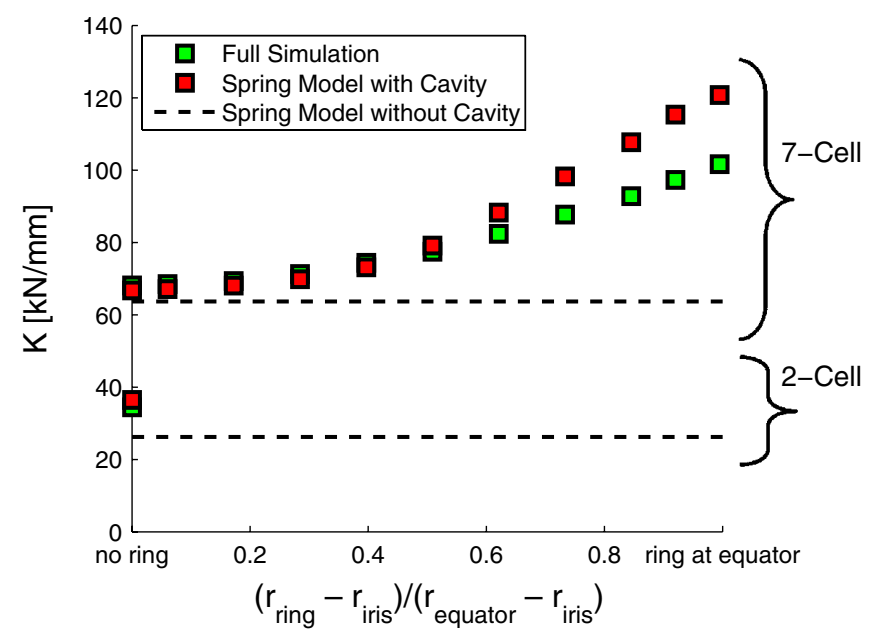

FIG. 10. $K$ of the two cavity assemblies, with data from both a simulation of the full model and from simulations of individual parts, put together using the equations in Fig. 9.
TABLE III. Percent reduction in overall stiffness of the He tank-tuner assembly as a result of a $50 \%$ reduction in the stiffness of one of its parts.

\begin{tabular}{lcc}
\hline \hline Part undergoing & $\begin{array}{c}\text { Reduction in } \\
\text { stiffness of 7-cell } \\
\text { He tank-tuner } \\
\text { reduction }\end{array}$ & $\begin{array}{c}\text { Reduction in } \\
\text { stiffness of 2-cell } \\
\text { He tank-tuner } \\
\text { assembly }\end{array}$ \\
\hline Bellows & $0.9 \%$ & $1.1 \%$ \\
Tuner dish & $0.0 \%$ & $9.5 \%$ \\
Coupler dish & $28 \%$ & $7.3 \%$ \\
Tuner & $37 \%$ & $44 \%$ \\
\hline \hline
\end{tabular}

For both the 2-cell and the 7-cell cavities, the largest contribution to $d F / d p$ is the helium pressure on the end walls of the helium tank, pushing the cavity apart. This is shown in the ANSYS simulation results in Fig. 11. The inset picture illustrates the two types of simulations that were run. In the first, pressure was applied to both the green and red areas and the restoring force on the cavity ends was found. In the second, the pressure was removed from the red areas, which make up the end walls.

There is some contribution from the pressure on the cavity, but removing the pressure from the end walls decreases the magnitude of $d F / d p$ by at least a factor of 4. Naively, one may predict the ratio of 2-cell assembly's $d F / d p$ to that of the 7-cell to be approximated by the ratio of the end wall areas (from beam tube OD to helium tank ID). However, this ratio, 0.96, is not in agreement with the $d F / d p$ ratio of 2.3 found in the simulations. The apparent discrepancy is caused by the setup of the tuners. In the 2-cell setup, the pressure on the end walls acts fully on the parallel combination of the cavity and the tuner. However, in the 7-cell setup, on the tuner side, only the pressure on

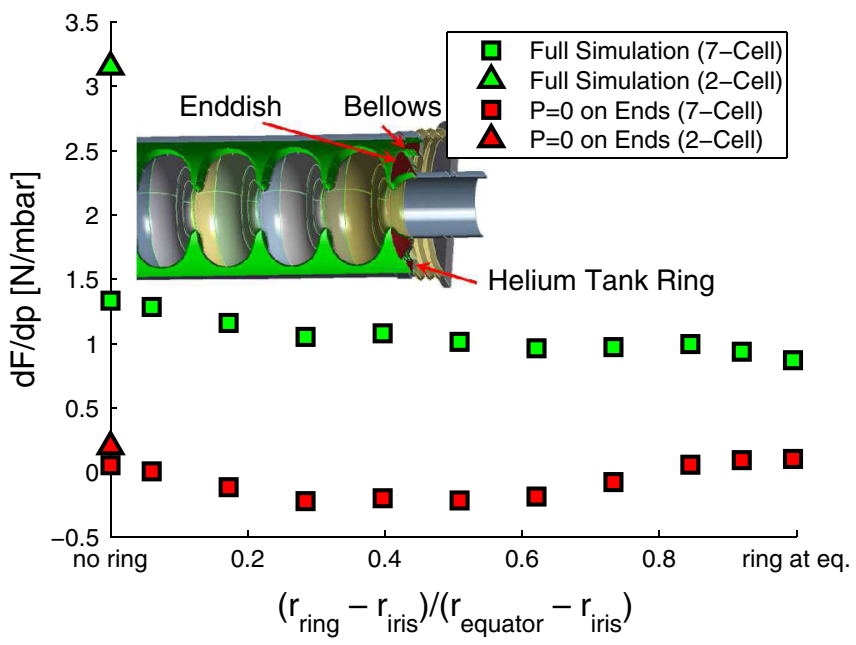

FIG. 11. $d F / d p$ of the cavities, showing both the nominal result and the result if no pressure is applied to the end walls. In the latter case, the magnitude of $d F / d p$ decreases nearly to zero. 


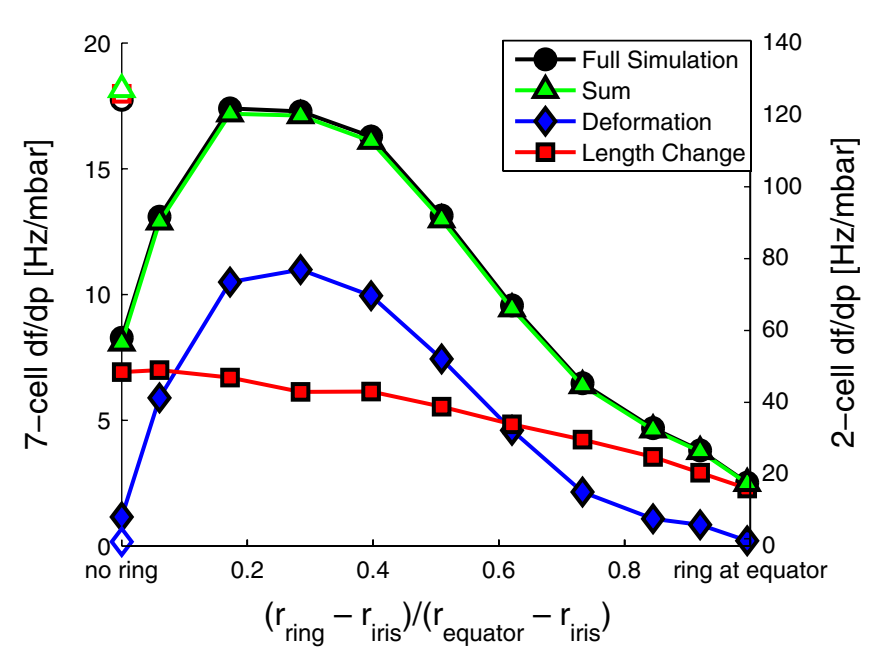

FIG. 12. Comparison of the breakdown of $d f / d p$ to the full simulation. The open markers correspond to the 2-cell cavity.

the enddish acts on the cavity and the tuner. Because the helium tank ring (indicated in Fig. 11) is separated from the enddish by a bellows, the pressure on it acts entirely on the helium tank, which is very rigid, so the effect of this pressure on the cavity length is negligible. Subtracting the area of the tank ring from the 7-cell's end wall area gives a 2 -cell to 7 -cell ratio of 1.9 , which is much closer to the simulated ratio.

The product of all these factors gives the contribution of length change to $d f / d p$. This is shown, with the additional cell shape change contribution in Fig. 12. The sum of the two is compared to the full simulation.

The breakdown of the models matches up very closely to the full simulation. The difference between the two is less than $3 \%$ for every point. This shows that methods for increasing $K$ or reducing $d f_{\text {shape }} / d p, d f / d L$, or $d F / d p$ should all be valid approaches for reducing $d f / d p$.

For both the 2-cell and the unstiffened 7-cell cavities, the deformation of the cell shape at fixed length changes the frequency very little. The positive frequency shift from the equator deformation balances the negative shift from the iris deformation. For the 7-cell, the variation in $d f / d p$ with stiffening ring radius is caused mostly by the way the rings shift this balance. Across the range of ring radii, the shape component of $d f / d p$ varies by a factor of 9.5, whereas the length component varies by only 3.3.

\section{METHODS TO DECREASE $d f / d p$}

Beyond proper design of the stiffening rings, some effective methods for decreasing $d f / d p$ are evident from the simulations and the model discussed above. Simple steps can be taken to reduce the contribution from length change, which is the largest factor for both the 2-cell and for the 7-cell with optimized stiffening rings. As presented above, using a tuner on the cavity end instead of a tuner on top of the helium tank decreases the effective area acted

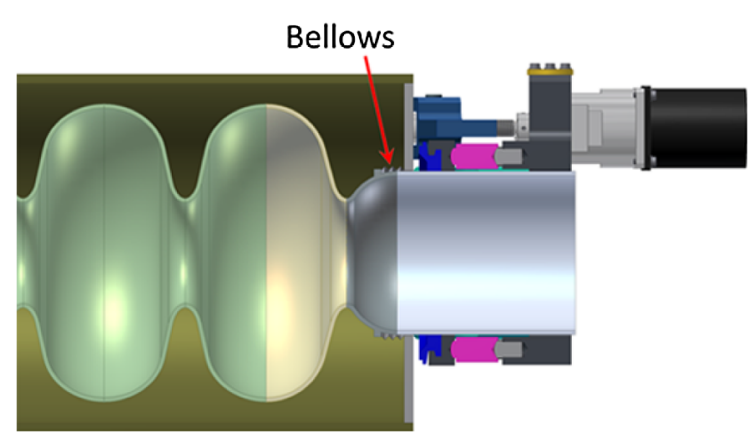

FIG. 13. One method of decreasing $d f / d p$ is reduction of the diameter of the bellows used in conjunction with the Saclay tuner.

upon by the helium pressure, thereby decreasing $d F / d p$ strongly. In addition, ensuring that both the tuner and the end walls are very stiff will also significantly decrease $d f / d p$.

As others (see, for example, [24]) have suggested before, it is possible to minimize $d f / d p$ in a cavity using a tuner located at the cavity end by optimizing the diameter of the helium tank bellows. Reducing the diameter of the bellows lowers the effective surface area of the end wall, i.e., the part which is rigidly connected to the cavity and creates a force that stretches the cavity when the helium pressure increases. As a result, $d F / d p$ decreases. A method to extend this to tuners that sit on top of the helium tank has also been suggested [25].

Using the unstiffened 7-cell cavity as an example, increasing the stiffness of the end wall opposite the tuner by a factor of 3 would increase the overall stiffness by $32 \%$. This could be accomplished using stiffening ribs. Decreasing the diameter of the bellows from 191 to $118 \mathrm{~mm}$ - an extreme case, as shown in Fig. 13-would reduce $d F / d p$ by a factor of 4.4 . Because the stiffness of the end wall also affects $d F / d p$, implementing these changes together reduces the length change of the cavity by an overall factor of 7.6. Assuming $d f_{\text {shape }} / d p$ and $d f / d L$ stay the same, $d f / d p$ would be reduced from $8.3 \mathrm{~Hz} / \mathrm{mbar}$ to just $2.9 \mathrm{~Hz} / \mathrm{mbar}$.

\section{CONSEQUENCES OF STIFFENING RING OPTIMIZATION}

The approach to $d f / d p$ reduction by optimization of the stiffening rings led to the promotion of no/small stiffening rings or large stiffening rings. However, the radius of the stiffening rings will have an impact in several areas other than microphonics.

\section{A. Cavity tuning}

While fine tuning of the cavity in the cryomodule is accomplished via piezoelectric actuators, a mechanical tuner is required to provide coarse tuning with a typical range of $\sim 500 \mathrm{kHz}$. The results of ANSYS calculations of 


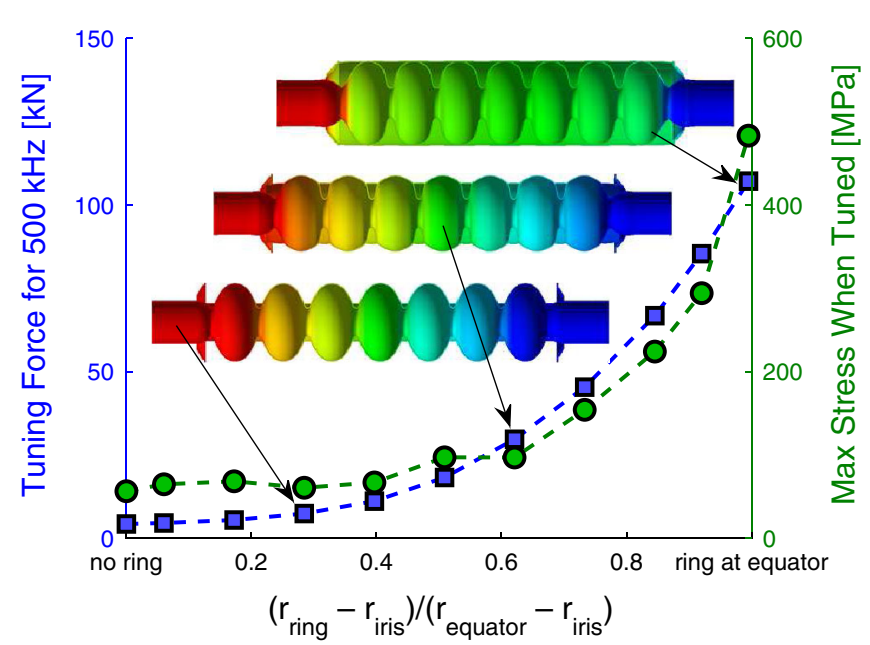

FIG. 14. Required force for frequency tuner and maximum stress in the cavity at maximum tuning. Inset are images showing deflection in the cavity for selected stiffening ring radii. Blue locations are least deflected, and red locations are most deflected.

the force required to provide this tuning via changing the length of the cavity are plotted in Fig. 14. Inset are plots showing the deflection of the cavity.

At the largest stiffening ring radii $(>80 \%$ of the irisequator distance), the force required from the tuner would be very large $(>60 \mathrm{kN})$ which would make the frequency tuner design very challenging. Moreover, eventually the cavity becomes stiffer than the enddishes, at which point the deflection in the middle cells is small relative to the deflection in the end cells. In this case, the tuner would only significantly affect the endcells, making it impossible to tune the cavity while maintaining field homogeneity in the cells.

The stress in the cavity at maximum tuning $(500 \mathrm{kHz})$ for any of these cases is high, and above the room temperature yield strength of roughly $50 \mathrm{MPa}$. Fortunately, due to changes in the material properties with temperature, the maximum allowable stress at $4.2 \mathrm{~K}$ is significantly higher, close to $140 \mathrm{MPa}$ [26], which sets the limit on the radius of stiffening rings at approximately $70 \%$ of the iris-equator distance. However, in any case, it is very important to set interlocks on the tuner that will release the stress if the cryomodule is warmed up. Failure to release the stress on the cavity when transitioning from cold to warm could result in plastic deformation.

\section{B. Deformations during handling}

A cavity with no/small stiffening rings can be very sensitive to small forces generated by its own weight and by handling. Figure 15 shows ANSYS results of a cavity deforming under its own weight, while supported only at the bottom of its enddishes. For small rings, the maximum stress in the cavity approaches the room temperature yield strength. In this case, great care must be taken to ensure the

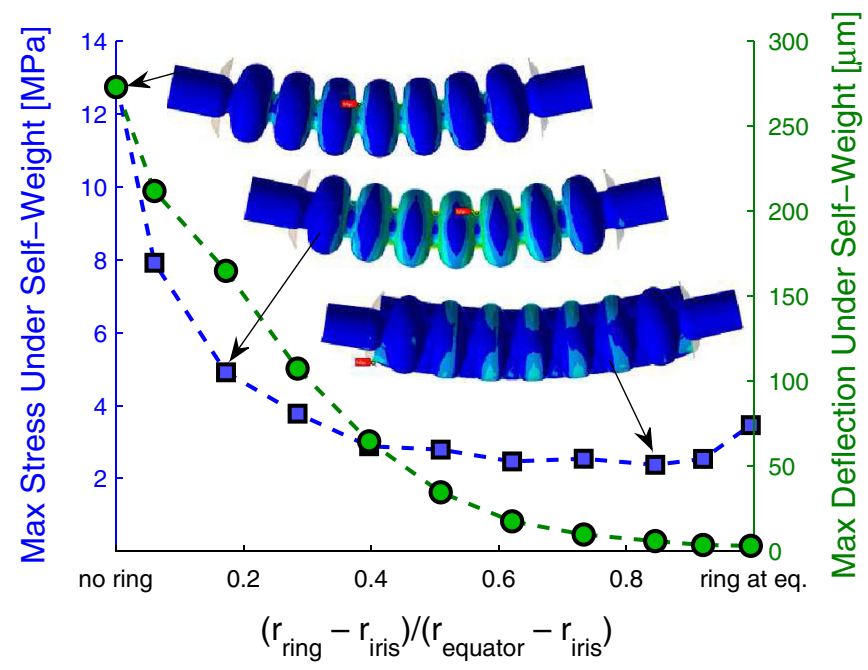

FIG. 15. Effect of gravity on a cavity seated on its enddishes.

cavity is properly supported at all times to avoid undesired deformation. A strong support frame can help.

Researchers at Jefferson Lab recently built cavities both with and without stiffening rings. They report a stronger tendency for unstiffened cavities to deform during handling, observed as a deterioration of the field flatness [27].

\section{Effect of mechanical resonances on active compensation}

Mechanical resonances of cavities in a cryomodule can be excited, for example, by external vibrations, helium bath pressure fluctuations, or by Lorentz forces in pulsed operation. Stiffening rings strongly affect the frequency of the mechanical resonances of the cavity. ANSYS calculation of the frequencies of the first six eigenmodes in the ERL 7-cell cavity are shown in Fig. 16 along with some illustrations of these modes. These results assume a $100 \mathrm{kN} / \mathrm{mm}$ tuner stiffness.

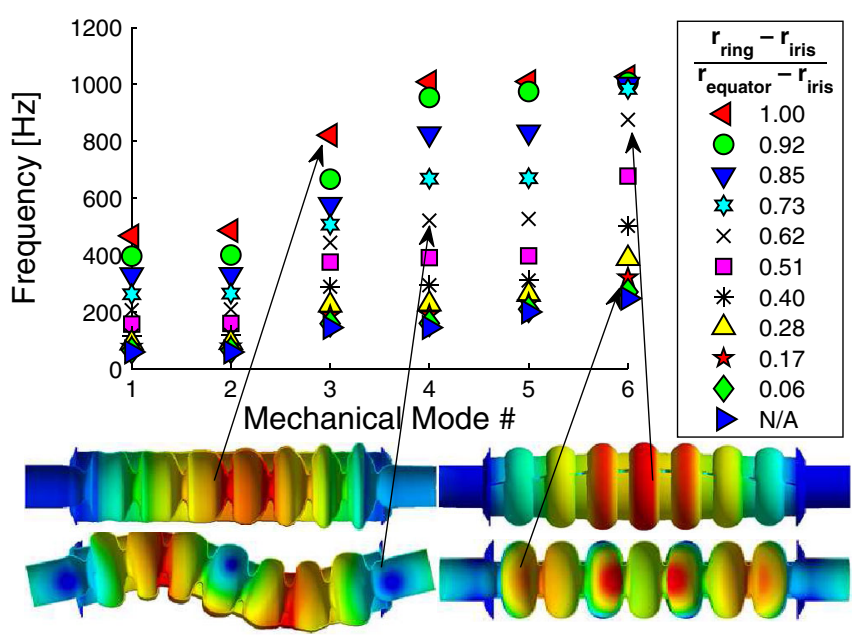

FIG. 16. Frequencies of the first 6 mechanical resonances of the 7 -cell cavity for various stiffening ring radii. 
From Fig. 16, it can be concluded that larger stiffening rings have mechanical modes at significantly higher frequencies, which is beneficial for two reasons. First, it makes active compensation of microphonics via the piezofrequency tuner more effective; second, the amplitudes of vibration sources tend to be lower at higher frequencies. Assuming that the lowest frequency mode limits the control bandwidth and that it has a similar amplitude for no stiffening rings and for stiffening rings at $70 \%$ of the irisequator distance, the latter case would have a control bandwidth 3.5 times larger than that of the former.

\section{Difficulty in fabrication}

Stiffening rings contribute significantly to the fabrication cost of an SRF cavity, so a cavity without rings is preferable from a cost point of view.

\section{E. Lorentz force detuning}

LFD was simulated in ANSYS, again using a procedure similar to the $d f / d p$ simulations, but with a pressure load calculated from the Lorentz forces from fields in the cavity instead of a constant pressure. A spring boundary condition is applied between the two ends with spring coefficient selected to simulate the helium tank-tuner assembly. The results are shown in Fig. 17 for the 2-cell, the 7-cell, and for an ILC cavity.

The average of the LFD coefficients for the five injector cavities was measured with the cavities on resonance, as shown in Fig. 18. The average is shown in Fig. 17 with error bars derived from the large spread in values from cavity to cavity. The results show good agreement between simulations and measurement. As expected, the ILC ring radius gives a low LFD coefficient. On the other hand, ring radii optimized for low $d f / d p$ have relatively high LFD

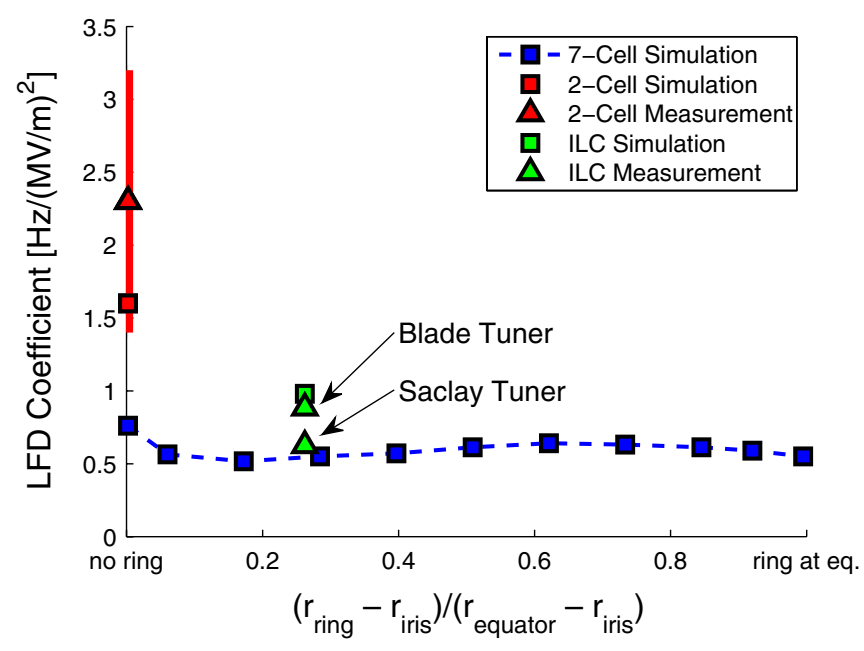

FIG. 17. Lorentz force detuning coefficients from ANSYS of the ERL injector and main linac cavities. The ILC cavity is also shown for comparison, with measurement reported from [20].
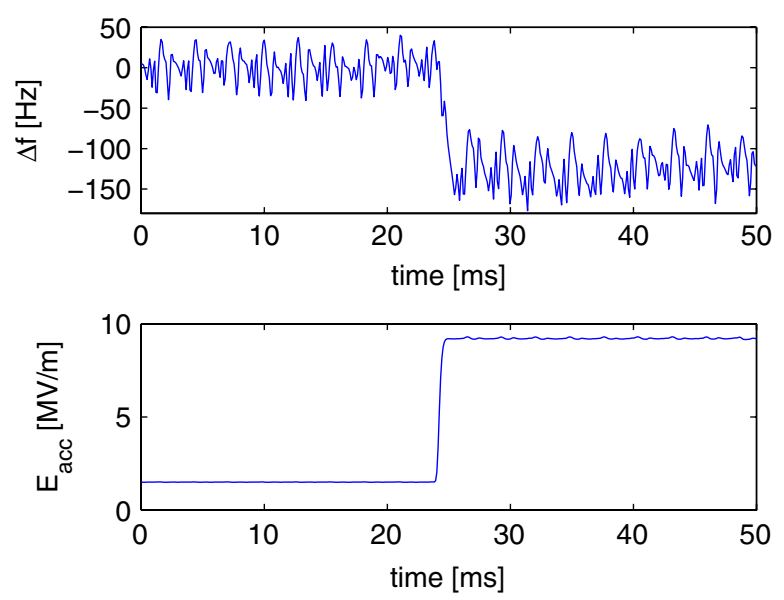

FIG. 18. Lorentz force detuning measurement of an ERL injector cavity.

coefficients, but this is not an issue for a cavity in $\mathrm{cw}$ operation.

\section{CONCLUSIONS}

Continuous wave superconducting rf linacs with no or low beam loading require operating their SRF cavities at high loaded quality factors to reduce costs and maximize operational efficiency. However, high loaded quality factors must be accompanied by low microphonics detuning levels. Therefore cavities need to have small sensitivity $d f / d p$ to fluctuations in the liquid helium bath pressure surrounding the cavity. In this paper, several methods were presented to significantly reduce $d f / d p$ in elliptical cavities through optimization of the cavity-helium tank-tuner system. Constructing a cavity with no/small radius stiffening rings or with large radius stiffening rings decreases $d f / d p$ from shape deformations. Using a tuner located at the end of the cavity instead of in the middle of the helium tank reduces $d f / d p$ from length change by decreasing the force generated by the pressure acting on the end walls. This effect can be further exploited by using as small a diameter as possible for the bellows between the helium tank ring and the enddish. In addition, ensuring that the tuner and the coupler enddish are as stiff as possible significantly decreases $d f / d p$ from length change. For the Cornell ERL main linac cavity, simulations show that implementing all of these methods, including using a cavity with no stiffening rings, would yield a $d f / d p$ of just $2.9 \mathrm{~Hz} / \mathrm{mbar}$. Compare this to measurements of the $d f / d p$ of a TTF cavity, approximately $30 \mathrm{~Hz} / \mathrm{mbar}$. Large, state of the art cryogenic systems achieve rms pressure stabilities of $\sim 0.1$ mbar [28]. Assuming a peak deviation 6 times the rms value, the peak detuning of the TTF cavity would be $18 \mathrm{~Hz}$, which gives an optimized $Q_{L}$ of $4 \times 10^{7}$, compared to $1.7 \mathrm{~Hz}$ and $4 \times 10^{8}$, respectively, for the optimized ERL main linac cavity. For operation at $13 \mathrm{MV}$ per cavity, this corresponds to a power requirement 
of $3.0 \mathrm{~kW}$ for a TTF cavity (scaled to 7 cells), and only $0.3 \mathrm{~kW}$ for the optimized ERL cavity.

For the Cornell ERL main linac cavity, two prototypes are being fabricated to investigate the two optimized stiffening ring radii solutions, one with no stiffening rings, and one with large stiffening rings, at approximately $70 \%$ of iris-equator distance. The bellows diameter was chosen as small as possible to accommodate the large stiffening rings. The unstiffened cavity will be easier to tune and easier to manufacture than the stiffened cavity, but it will be more fragile during handling and its low frequency mechanical resonances will limit the bandwidth of the piezotuner. The decision of whether or not to use stiffening rings will be based on the experience of fabrication and operation under horizontal test.

\section{ACKNOWLEDGMENTS}

The authors would like to express their thanks to Vadim Veshcherevich, Roger Kaplan, John Dobbins, and Peter Quigley for help with measurements on the ERL injector, as well as to Rick Fischer from ANL for his helpful advice on interfacing ANSYS Workbench with APDL. This work was supported by NSF Award No. DMR-0807731.

[1] J. Holzbauer, W. Hartung, and J. Popielarski, in PAC 2011, New York, NY (PAC'11 OC/IEEE, New York, 2011), TUP090.

[2] E. Zaplatin et al., SRF 2009, Berlin, Germany (Helmholtz Zentrum Berlin, Berlin, Germany, 2009), THPPO013.

[3] L. Ristori et al., in PAC 2011, New York, NY (Ref. [1]), TUP084.

[4] Z. Conway et al., in Proceedings of the 12th International Workshop RF Superconducting (SRF2005), Ithaca, NY, TUA06 [http://www.lepp.cornell.edu/public/SRF2005/].

[5] A. Facco, in Proceedings of the 12th International Workshop RF Superconducting (SRF2005), Ithaca, NY [http://www.lepp.cornell.edu/public/SRF2005/].

[6] H. Sakai et al., at the ERL09: Energy Recovery Linac, 45th ICFA Beam Dynamics Workshop, 2009, Cornell University.

[7] N. Solyak et al., in Proceedings of the IPAC'10 Conference, Kyoto, Japan (ICR, Kyoto, 2010), MOPEC081.

[8] W. Schappert et al., PAC 2011, New York, NY (Ref. [1]), TUP086.

[9] V. Yakolev et al., PAC 2011, New York, NY (Ref. [1]), TUP086.
[10] M. Liepe et al., in Proceedings of the 23rd Particle Accelerator Conference, Vancouver, Canada, 2009 (IEEE, Piscataway, NJ, 2009), TU3RAI01.

[11] C. Pagani, ILC Workshop 2005, Snowmass, Colorado (2005).

[12] N. Valles and M. Liepe, in Proceedings of the XXV Linear Accelerator Conference (LINAC 10), Tsukuba, Japan (High Energy Accelerator Research Organization (KEK), Japan, 2010) [http://spms.kek.jp/pls/linac2010/ TOC.htm].

[13] V. Shemelin et al., in Proceedings of the 20th Particle Accelerator Conference, Portland, OR, 2003 (IEEE, New York, 2003), pp. 2059-2061.

[14] M. Liepe et al., SRF 2009, Berlin, Germany (Ref. [2]), MOOBAU04.

[15] D. Barni, A. Bosotti, and C. Pagani, in Proceedings of the 8th European Particle Accelerator Conference, Paris, 2002 (EPS-IGA and CERN, Geneva, 2002), THPDO024.

[16] http://www.ANSYS.com.

[17] S. Posen and M. Liepe, SRF 2011, Chicago, IL (2011), THPO066.

[18] K. M. Wilson et al., in Proceedings of the 20th Particle Accelerator Conference, Portland, OR, 2003 (Ref. [13]), RPAB064.

[19] W. Hartung et al., in Proceedings of the 18th Particle Accelerator Conference, New York, 1999 (IEEE, New York, 1999).

[20] W.-D. Möller and M. Pekeler, in Proceedings of EPAC 96 (1997), Vol. 1, p. 1048 [http://accelconf.web.cern.ch/ accelconf/e96/PAPERS/MOPG/MOP085G.PDF].

[21] O. Kugeler et al., in Proceedings of the 10th European Particle Accelerator Conference, Edinburgh, Scotland, 2006 (EPS-AG, Edinburgh, Scotland, 2006), MOPCH149.

[22] D. A. Edwards et al., "TESLA Test Facility Linac Design Report," DESY, Chap. 4, 1995.

[23] Small differences in cell shape in both the 7-cell (end cells compared to the center cells) and the 2-cell (first compared to the second cell) will create a second order perturbation on this factor.

[24] E. Zaplatin et al., in in Proceedings of the 10th European Particle Accelerator Conference, Edinburgh, Scotland, 2006 (Ref. [21]), MOPCH157.

[25] E. Zaplatin, PAC 2011, New York, NY (Ref. [1]), TUP031.

[26] G. Wu, H. Edwards, and T. Peterson, Fermilab note, Fermi National Accelerator Laboratory, Batavia, IL, 2008.

[27] F. Marhauser, Jefferson Laboratory, Report No. JLab-TN10-021, 2010.

[28] J. Weisend (private communication). 